\title{
Observation of coupled-cavity structures in metamaterials
}

Humeyra Caglayan, Irfan Bulu, Marko Loncar, and Ekmel Ozbay

Citation: Appl. Phys. Lett. 93, 121910 (2008);

View online: https://doi.org/10.1063/1.2988286

View Table of Contents: http://aip.scitation.org/toc/apl/93/12

Published by the American Institute of Physics

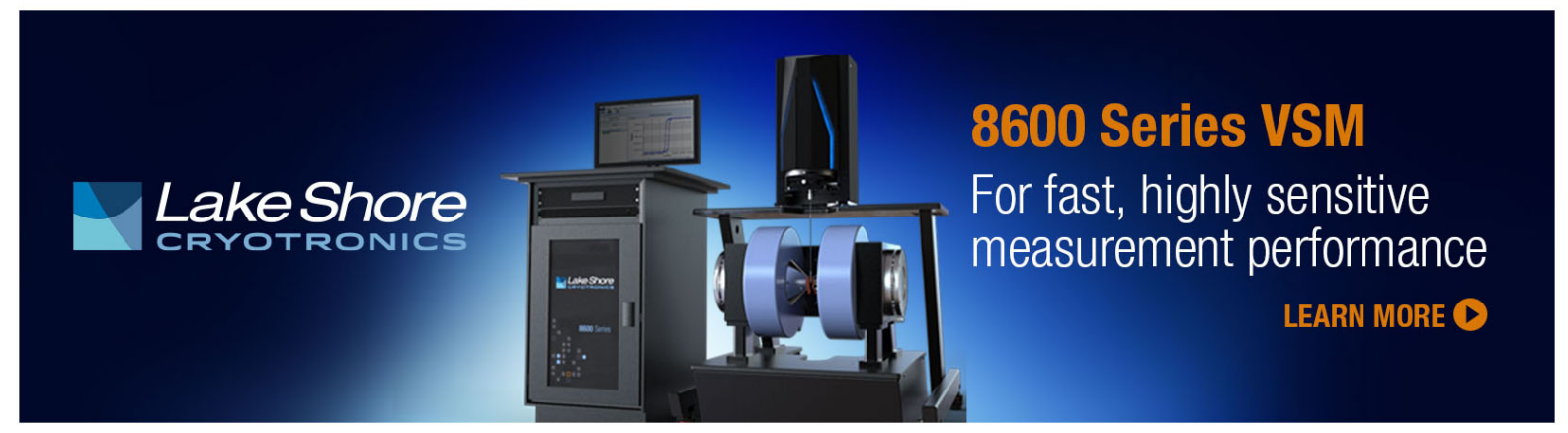




\title{
Observation of coupled-cavity structures in metamaterials
}

\author{
Humeyra Caglayan, ${ }^{1, a)}$ Irfan Bulu, ${ }^{2}$ Marko Loncar, ${ }^{2}$ and Ekmel Ozbay ${ }^{1}$ \\ ${ }^{1}$ Nanotechnology Research Center-NANOTAM, Department of Physics, and Department of Electrical and \\ Electronics Engineering, Bilkent University, Bilkent, 06800 Ankara, Turkey \\ ${ }^{2}$ School of Engineering and Applied Sciences, Harvard University, 33 Oxford Street, Cambridge, \\ Massachusetts 02138, USA
}

(Received 9 July 2008; accepted 2 September 2008; published online 25 September 2008)

\begin{abstract}
In this letter, we investigated the transmission properties of metamaterial based coupled-cavity structures. We first calculated the effective parameters of a split-ring resonator (SRR) and composite metamaterial (CMM) structures. Subsequently, we introduced coupled-cavity structures and presented the transmission spectrum of SRR and CMM based coupled-cavity structures. The splitting of eigenmodes was observed due to the interaction between the localized electromagnetic cavity modes. Finally, the dispersion relation and normalized group velocity of the coupled-cavity structures were calculated. The maximum group velocity was found to be 100 times smaller than the speed of light in vacuum. (C) 2008 American Institute of Physics. [DOI: 10.1063/1.2988286]
\end{abstract}

Left-handed materials have recently attracted much attention in the scientific community. This new type of artificial materials can provide simultaneous negative permittivity $(\varepsilon)$ and permeability $(\mu)$ over a certain frequency range. Therefore, these materials possess promising physical properties and applications, such a negative refraction ${ }^{1}$ and subwavelength focusing. ${ }^{2}$ Negative permittivity is available through metals or the periodic arrangement of metallic wires. ${ }^{3}$ On the other hand, obtaining negative permeability was an issue because of the lack of a magnetic charge. However, in 2002, Pendry et al. proposed split-ring resonators (SRRs) in order to obtain negative permeability. ${ }^{4}$ Inspired by encouraging ideas of Pendry et al. for obtaining negative permittivity and negative permeability at microwave frequencies, Smith et al. succeeded in designing and demonstrating a composite metamaterial (CMM) with a negative index of refraction. ${ }^{5}$ Electromagnetic waves cannot propagate in the negative $\mu$ or negative $\varepsilon$ medium. Therefore, the SRR (CMM) structure exhibits a gap in the spectrum for the negative $\mu(\varepsilon)$ region. Hence, it is possible to obtain highly localized cavity modes inside the stop band by introducing a defect into the structure, which is similar to the defect modes in photonic crystals (PCs).

In our previous paper, we studied CMM based single cavity system. ${ }^{6}$ We have reported that it is possible to obtain cavity mode in the transmission spectrum with high quality factors. In the present work, we investigated the transmission properties of SRR and CMM based coupled-cavity structures with a different cavity structure. First of all, we calculated the effective parameters of SRR and CMM structures. We introduced coupled-cavity structures and presented the transmission spectrum of SRR and CMM based coupled-cavity structures. Subsequently, by using the tight-binding (TB) approach, we calculated the dispersion relation and normalized group velocity of the coupled-cavity structures.

The SRR structure that we used for the present study was a one-dimensional periodic arrangement of square rings. The CMM structure was obtained by the combination of the SRR structure and wire stripes. The wire stripes were on the

${ }^{\text {a)} E l e c t r o n i c ~ m a i l: ~ c a g l a y a n @ f e n . b i l k e n t . e d u . t r . ~}$ back of the substrate and the square SRRs were on the front faces. The structures were printed on a Teflon substrate with $\varepsilon=2.17$, in which the thickness of the substrate was $1 \mathrm{~mm}$. The thickness of the copper was $0.05 \mathrm{~mm}$. The width of the wire stripes was $1.6 \mathrm{~mm}$. The lattice constant along the $x$ direction (propagation direction) was $4.95 \mathrm{~mm}$. The height of the structure was 40 unit cells. 30 layers of the structures were stacked with a $2 \mathrm{~mm}$ period along the $z$ direction. The E-field was in the $y$ direction. The details regarding the unit cell of the metamaterial structures are shown in Figs. 1(a) and $1(b)$.

We first calculated the effective $\varepsilon$ and $\mu$ of the SRR and CMM structures by use of a retrieval procedure. The retrieval procedure is widely used to calculate the effective parameters of the metamaterials. In this method, the real and imaginary parts of the refractive index, wave impedance, and, therefore, the real and imaginary parts of the $\varepsilon$ and $\mu$ are retrieved from the amplitude and the phase information of the transmission and reflection. The details of the retrieval procedure that were used in this study are outlined in Ref. 7. This particular method has the advantage of identifying the correct branch of the effective $\varepsilon$ and $\mu$. The ambiguity in the determination of the correct branch is resolved by the use of an analytic continuation procedure. There was one layer of the structure along the propagation direction in this calculation. We employed periodic boundary conditions along directions other than the propagation direction. Therefore, the simulation setup coincides with a slab of material that con-

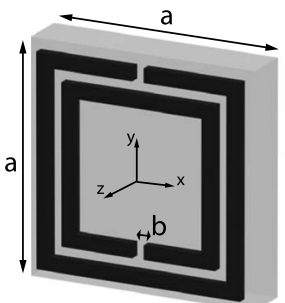

(a)

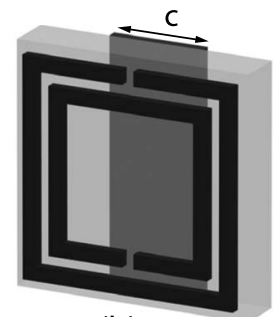

(b)

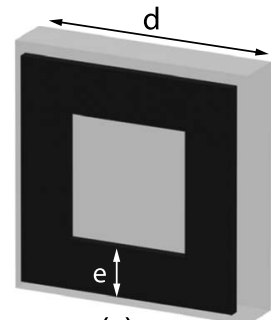

(c)
FIG. 1. (a) The unit cell of the SRR structure: $a=4.95 \mathrm{~mm}, b=0.25 \mathrm{~mm}$. (b) the unit cell of the CMM structure: $c=1.6 \mathrm{~mm}$. (c) the cavity structure: $d$ $=5.4 \mathrm{~mm}, e=1 \mathrm{~mm}$. The unit cells of metamaterials are much smaller than the operating wavelength. 


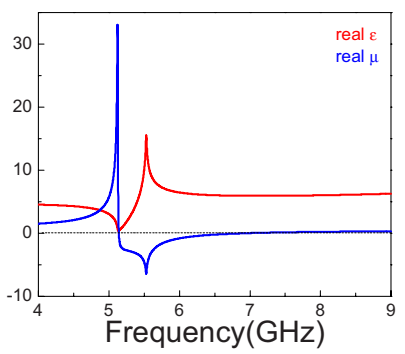

(a)

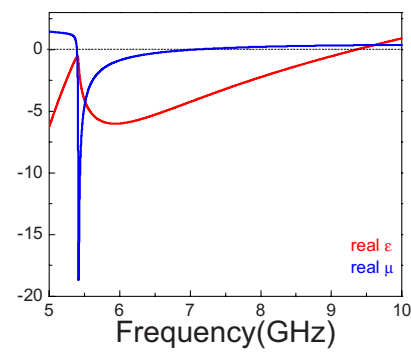

(b)
FIG. 2. (Color online) (a) The calculated parameters show that the SRR structure possesses effective $\varepsilon>0, \mu<0$ from 5.0 to $7.0 \mathrm{GHz}$. (b) The CMM structure possesses effective $\varepsilon<0, \mu<0$ from 5.4 to $7.0 \mathrm{GHz}$ and $\varepsilon<0, \mu>0$ from 7.0 to $9.4 \mathrm{GHz}$ [Fig. 2(b)].

sists of a single layer. The effective $\varepsilon$ and $\mu$ were derived from the transmission and reflection coefficients. The calculated parameters show that the SRR structure possesses effective $\varepsilon>0, \mu<0$ from 5.0 to $7.0 \mathrm{GHz}$ [Fig. 2(a)]. However, the CMM structure possesses effective $\varepsilon<0, \mu<0$ from 5.4 to $7.0 \mathrm{GHz}$ and $\varepsilon<0, \mu>0$ from 7.0 to $9.4 \mathrm{GHz}$ [Fig. 2(b)].

The measured transmission spectrum of the SRR exhibits a band gap between 5.0 and $7.0 \mathrm{GHz}$ [Fig. 3(a)]. On the other hand, the CMM structure transmits em waves at the band gap frequency of the SRR [Fig. 4(a)], since it has negative $\mu$ and $\varepsilon$ at this range. The simulation results agree well with the measurement. The experimental setup consisted of an HP 8510C network analyzer and two standard gain horn antennae in order to measure the transmission amplitude. The transmission simulations throughout the letter were performed by the commercial software program CST MICROWAVE STUDIO®.

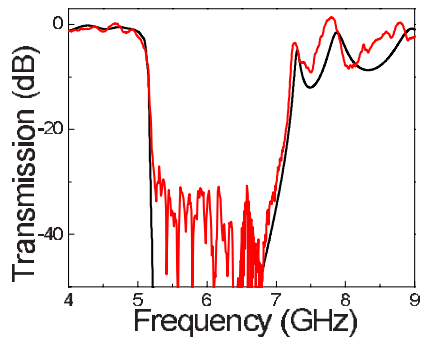

(a)

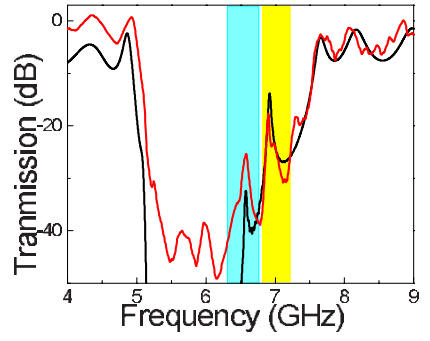

(c)

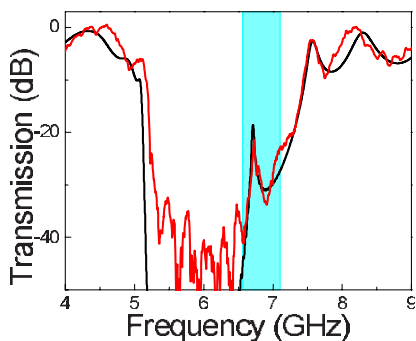

(b)

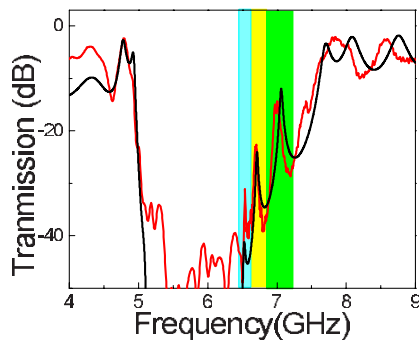

(d)
FIG. 3. (Color online) Measured (red curves) and calculated (black curves) transmission spectra for (a) SRR and (b) SRR with a single-cavity, (c) twocoupled-cavity, and (d) three-coupled-cavity structures. Due to the coupling between the strongly localized cavity modes, the single-cavity mode splits into two or three distinct modes depending on the number of coupled cavities. There is a good agreement between the measured and calculated transmission spectra.

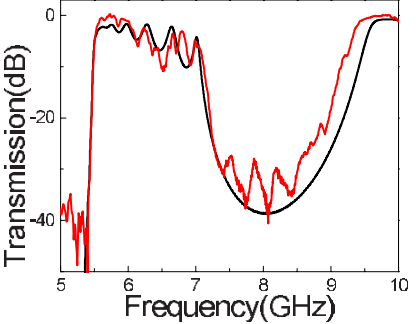

(a)

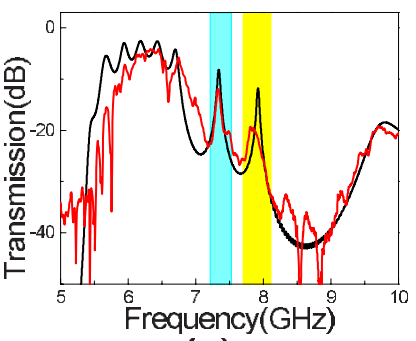

(c)

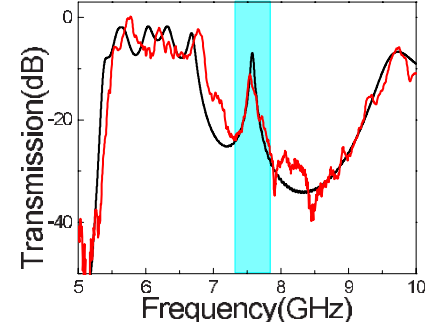

(b)

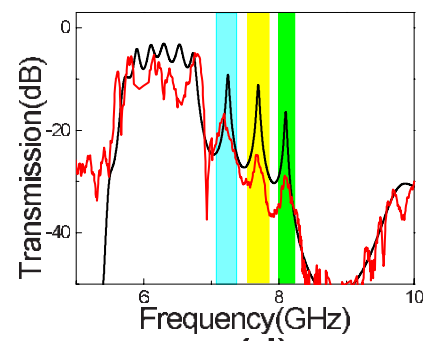

(d)
FIG. 4. (Color online) Measured (red curves) and calculated (black curves) transmission spectra for (a) CMM and (b) CMM with a single-cavity, (c) two-coupled-cavity, and (d) three-coupled-cavity structures.

Subsequently, we introduced the defect structure into the left-handed structures and we changed the center unit cell of the structures by a closed ring structure. The total singlecavity system contains one cavity at the center and two metamaterial unit cells in each side of the cavity ( 1 cavity +4 metamaterial unit cells) in the propagation direction. The defect structure is a closed ring structure, which was placed on both sides of the board and possessed positive $\varepsilon$ and $\mu$ [Fig. 1(c)]. In the presence of a single cavity, a localized cavity mode is observed in the transmission spectrum of SRR and CMM based structures. The measured cavity resonance frequencies are $\Omega=6.7 \mathrm{GHz}$ and $7.6 \mathrm{GHz}$ [Figs. 3(b) and $4(b)$.

Moreover, we brought two cavities together with an intercavity distance of two metamaterial unit cells. The total two-cavity system contains two cavities and two metamaterial unit cells in each side of the each cavity ( 2 cavities +6 metamaterial unit cells) in the propagation direction. For two coupled cavities, the transmission characteristics as a function of frequency are measured and calculated. As shown in Figs. 3(c) and 4(c), we observed that the resonance modes are split into two distinct symmetric and antisymmetric modes similar to PC cavity structure. In PC cavity structures, when two isolated cavities are brought together, the localized photon modes should overlap. Due to this interaction, the doubly degenerate eigenmode splits into two distinct modes as symmetric and antisymmetric. ${ }^{8}$ These modes are reminiscent of the bonding and antibonding states in solid state physics. For example, in the diatomic molecules, the interaction between the two atoms produces a splitting of the degenerate atomic levels into bonding and antibonding orbitals. ${ }^{9}$ The measured values of resonance frequencies are $\omega_{1}=6.5 \mathrm{GHz}$ and $\omega_{2}=6.9 \mathrm{GHz}$ for the SRR based cavity structure and $\omega_{1}=7.3 \mathrm{GHz}$ and $\omega_{2}=7.9 \mathrm{GHz}$ for the CMM based cavity structure.

When we brought three cavities together with an intercavity distance of two metamaterial unit cells, the single cavity mode $\Omega$ splits into three different eigenmodes. In this 
case, the total three-cavity system contains three cavities and two metamaterial unit cells in each side of the each cavity ( 3 cavities +8 metamaterial unit cells) in the propagation direction. The corresponding transmission spectra of the system were measured and calculated. As shown in Figs. 3(d) and 4(d), there is a good agreement between the measured and calculated transmission characteristics of the three coupled cavities. The resonance frequencies were $\Gamma_{1}=6.4 \mathrm{GHz}, \Gamma_{2}$ $=6.7 \mathrm{GHz}$, and $\Gamma_{3}=7.0 \mathrm{GHz}$ for the SRR based cavity structure and $\Gamma_{1}=7.2 \mathrm{GHz}, \Gamma_{2}=7.6 \mathrm{GHz}$, and $\Gamma_{3}=8 \mathrm{GHz}$ for the $\mathrm{CMM}$ based cavity structure.

Recently, the classical wave analog of the TB picture ${ }^{10}$ has successfully been applied to photonic structures. ${ }^{11}$ By using the direct implications of the TB picture, a novel propagation mechanism for photons along the localized coupled-cavity modes in PCs was proposed ${ }^{12}$ and demonstrated. ${ }^{13}$ In these structures, photons can hop from one tightly confined mode to the neighboring one due to the weak interaction between them. The same approach can be applied to metamaterial based coupled cavities. Using the TB picture, it is possible to obtain eigenvalues and eigenvectors corresponding to two- and three-coupled cavity structures. ${ }^{14}$ Hence, the dispersion relation and group velocity can be obtained, keeping only the nearest-neighbor coupling terms as

$$
\begin{aligned}
& \omega(k)=\Omega[1+\kappa \cos (k \Lambda)], \\
& \nu_{g}(k)=\nabla_{k} \omega_{k}=-\kappa \Lambda \Omega \sin (k \Lambda) .
\end{aligned}
$$

Here, $\kappa$ is a TB parameter that can be obtained from the splitting of the eigenmodes of two-coupled cavities, in which $\Omega$ is the frequency mode of the single cavity, and $\Lambda$ is the distance between the two-cavity structures (intercavity distance).

The calculated TB parameters are $\kappa=-0.05$ and $\kappa=-0.07$ for SRR and CMM based coupled cavities, respectively. We can obtain the dispersion relation of the coupled cavity structures by using these TB parameters and $\Lambda$ $=9.9 \mathrm{~mm}$ ( 2 metamaterial unit cells). Figure 5(a) shows the calculated dispersion relations $\omega(k)$. We also plotted the normalized group velocity corresponding to the coupled-cavity structures. As shown in Fig. 5(b), the group velocity has a maximum value of nearly one-hundredth the speed of light in vacuum, at the coupled-cavity band center, and vanishes at the band edges.

As a conclusion, the transmission properties of the coupled-cavity structures in one-dimensional metamaterials have been investigated. The splitting of eigenmodes due to the interaction between the localized electromagnetic cavity modes was observed. The results are very similar to the PC coupled-cavity systems. However, taking the advantage of the subwavelength dimension of the metamaterial system, it is possible to localize fields into subwavelength regions us-

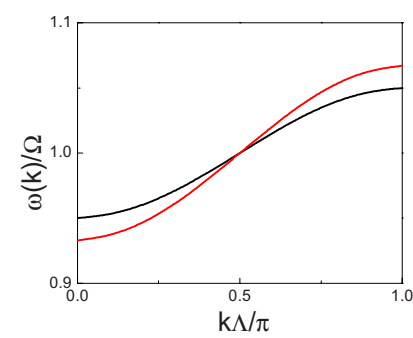

(a)

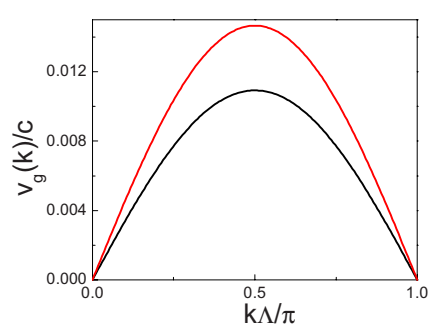

(b)
FIG. 5. (Color online) (a) The calculated dispersion relation of the coupledcavity structures is shown (black curves stand for the SRR based cavity and the red curves stand for the CMM cavity structure). (b) The group velocity is two orders of magnitude smaller than the speed of light at the band center $(k \Lambda=\pi / 2)$ and vanishes at the band edges $(k \Lambda=0$ and $\pi)$.

ing SRR and CMM based cavity systems. In taking advantage of the TB approach, we calculated the dispersion relation and group velocity of metamaterial based coupled-cavity structures. The group velocity was rather low for these structures. Therefore, the spontaneous emission rate and efficiency of the nonlinear processes can be enhanced in metamaterial based coupled-cavity structures.

This work is supported by the European Union under the projects EU-METAMORPHOSE, EU-PHOREMOST, EU-PHOME, and EU-ECONAM, and TUBITAK under the Project Nos. 105E066, 105A005, 106E198, and 106A017. One of the authors (E.O.) also acknowledges partial support from the Turkish Academy of Sciences.

${ }^{1}$ E. Ozbay and K. Aydin, Photonics Nanostruct. Fundam. Appl. 6, 108 (2008).

${ }^{2}$ I. Bulu, H. Caglayan, and E. Ozbay, Opt. Lett. 31, 814 (2006).

${ }^{3}$ J. B. Pendry, A. J. Holden, D. J. Robbins, and W. J. Stewart, J. Phys.: Condens. Matter 10, 4785 (1998).

${ }^{4}$ J. B. Pendry, A. J. Holden, D. J. Robins, and W. J. Stewart, J. Phys.: Condens. Matter 47, 2075 (1999).

${ }^{5}$ D. R. Smith, W. J. Padilla, D. C. Vier, S. C. Nemat-Nasser, and S. Schultz, Phys. Rev. Lett. 84, 4184 (2000).

${ }^{6}$ H. Caglayan, I. Bulu, M. Loncar, and E. Ozbay, Opt. Express 16, 11132 (2008)

${ }^{7}$ X. Chen, T. M. Grzegorczyk, B.-I. Wu, J. Pacheco, Jr., and J. A. Kong, Phys. Rev. E 70, 016608 (2004).

${ }^{8}$ M. I. Antonoyiannakis and J. B. Pendry, Phys. Rev. B 60, 2363 (1999).

${ }^{9}$ C. Kittel, Introduction to Solid State Physics (Wiley, New York, 1996).

${ }^{10}$ N. W. Ashcroft and N. D. Mermin, Solid State Physics (Saunders, Philadelphia, PA, 1976).

${ }^{11}$ E. Lidorikis, M. M. Sigalas, E. N. Economou, and C. M. Soukoulis, Phys Rev. Lett. 81, 1405 (1998).

${ }^{12}$ T. Mukaiyama, K. Takeda, H. Miyazaki, Y. Jimba, and M. KuwataGonokami, Phys. Rev. Lett. 82, 4623 (1999).

${ }^{13}$ M. Bayindir, B. Temelkuran, and E. Ozbay, Phys. Rev. Lett. 84, 2140 (2000).

${ }^{14}$ M. Bayindir, C. Kural, and E. Ozbay, J. Opt. A, Pure Appl. Opt. 3, 184 (2001) 